\title{
AN APPLICATION OF BANACH LINEAR FUNCTIONALS TO SUMMABILITY
}

\author{
BY \\ ALBERT WILANSKY
}

1. A summability matrix is called conservative if it attaches a limit to, that is, sums, every convergent sequence. If moreover this limit is a fixed multiple $m$ of the ordinary limit of the sequence the matrix is called multiplicative $m$. If a matrix $A$ is multiplicative $m$ then the matrix $k A$, where $k \neq 0$ is a number, is multiplicative $\mathrm{km}$ and sums exactly the same sequences as $A$. Thus it is immaterial to specify $m$ except to say whether or not it is zero. The dichotomy of matrices into those for which $m=0, m \neq 0$ is well known to be significant. A single example is the theorem of Steinhaus [1] $\left.{ }^{1}\right)$ that if $m \neq 0$ a multiplicative $m$ matrix cannot sum all bounded sequences, the result being false if $m=0$.

The principal object of this paper is to extend this classification into the whole set of conservative matrices. This will be separated into the subclasses of co-regular and co-null matrices; the division of multiplicative matrices induced will be that mentioned above. It will then be shown that a class of theorems which have been proved about multiplicative matrices can be so generalized as to apply to conservative matrices in general. The value of the classification will appear in that certain results in which the condition $m \neq 0$ plays an essential role will hold for co-regular but not for co-null matrices. Some results are new even for multiplicative matrices, for example, the specialization of Theorem 2.0.3.

1.1. Preliminary. Let $A=\left(a_{n k}\right)$ be a matrix of complex numbers and $x=\left\{x_{n}\right\}$ a complex sequence, then $y=A x$ is called the transformed sequence where in the multiplication $x$ and $y$ are treated as column vectors, thus $y=\left\{y_{n}\right\}$ where $y_{n}=\sum_{k=0}^{\infty} a_{n k} x_{k}=A_{n}(x)$. Then, if it exists, $\lim y_{n}=\lim A_{n}(x)$ is called $A(x)$. Finally the domain of the functional $A(x)$, that is, the set of sequences $x$ such that $A x$ is convergent, is called the summability field of $A$ and written $(A)$. We shall denote the identity matrix by $I$ so that $(I)$ is the set of convergent sequences.

Setting, after Brudno (1), $\|A\|=\sup _{n} \sum_{k=0}^{\infty}\left|a_{n k}\right|$ and denoting by $F$ the set of sequences $\dot{i}=\{1,1,1, \cdots\}, \delta^{0}=\{1,0,0, \cdots\}, \delta^{1}=\{0,1,0, \cdots\}$, $\cdots, \delta^{k}, \cdots$, we have the classical result: the matrix $A$ is conservative if and only if (i) $\|A\|<\infty$ and (ii) $F \subseteq(A)$.

Denoting $A\left(\delta^{k}\right)=\lim _{n \rightarrow \infty} a_{n k}$ by $a_{k}$ we can easily show that

Presented to the Society, April 26, 1947; received by the editors October 16, 1947, and, in revised form, June $30,1948$.

(1) Numbers in brackets refer to the bibliography at the end of the paper. 


$$
\sum\left|a_{k}\right|<\infty
$$

for a matrix satisfying (i) and (ii). It is well known that a conservative matrix $A$ is multiplicative if and only if

$$
a_{k}=0
$$

for each $k$.

A triangular matrix has $a_{n k}=0$ if $k>n$, while a triangular matrix for which $a_{n n} \neq 0$ for each $n$ is called normal.

We metrize $(A)$ by setting $\|x\|=\sup _{n}\left|\sum_{k=0}^{\infty} a_{n k} x_{k}\right|$. It is indicated in Mazur [2, p. 42] that if $A$ is normal and $\|A\|<\infty$ then $(A)$ is a Banach space using this norm; it is also shown that under the same conditions, if $\left\{x^{r}\right\}$ is a sequence of elements of $(A)$ with limit $x$, that is, $\left\|x^{r}-x\right\|$ tends to zero, then

$$
\lim _{r \rightarrow \infty} x_{k}^{r}=x_{k}
$$

for each $k$.

LemMA 1.1.1. If $A, B$ are conservative matrices with $(A) \subseteq(B)$ and $A$ is normal, then the metric of $A$ is stronger (in the sense of Banach [1, p. 41, Theorem 6]) than that of $B$ where both are defined. If $(A)=(B)$ and both $A$ and $B$ are normal the metrics are equivalent.

Setting $C=B A^{-1}$ we have $\left|B_{n}(x)\right|=\left|\sum_{k=0}^{\infty} x_{k} \sum_{r=k}^{\infty} c_{n r} a_{r k}\right|=\mid \sum_{r=0}^{\infty} c_{n r}$ $\cdot \sum_{k=0}^{r} a_{r k} x_{k} \mid \leqq\|x\|_{A} \cdot\|C\|$ and so $\|x\|_{B} \leqq\|x\|_{A} \cdot\|C\|$ and since, as it is easy to show, $C$ is conservative we have $\|C\|<\infty$. The result follows.

LEMMA 1.1.2. The summability field of an arbitrary matrix is separable.

Calling the matrix $A$ we are using the metric defined above. The norm preserving correspondence $x \leftrightarrow A x, x \in(A)$, between $(A)$ and the subset $J$ of (I) consisting of those sequences expressible as $A x, x \in(A)$, is $1-1$ if we identify members of $(A)$ whose distance apart is zero. Since $(I)$ is well known to be separable the conclusion follows.

If $A$ is normal let $A^{-1}=\left(a_{n k}^{\prime}\right)$ be the inverse matrix, let $a^{\prime k}$ denote the sequence whose $n$th term is $a_{n k}^{\prime}$, that is, the $k$ th column of $A^{-1}$, and let $s$ or $s_{A}$ denote the sequence whose $n$th term is $\sum_{k=0}^{n} a_{n k}^{\prime}$. Clearly $A_{n}\left(a^{\prime k}\right)=\delta_{n k}$, $A_{n}(s)=1$ and so these sequences belong to $(A)$. Mazur [2] uses the symbols $a^{(r)}, a ;$ p. 42 .

1.2. We make constant reference to the following theorems of Mazur. (A $B$-linear or Banach linear functional is an additive continuous functional.)

MAZUR'S THEOREMS. Let $A$ be a normal, conservative matrix; let $B$ be an arbitrary matrix. Then

(i) if $B \supseteq(A), B(x)$ is a B-linear functional on $(A)$,

(ii) if $L(x)$ is a B-linear functional on $(A)$ it is given by $L(x)=t A(x)$ $+\sum_{r=0}^{\infty} t_{r} A_{r}(x)$ where $\sum\left|t_{r}\right|<\infty$.

The representation is unique; indeed $t=t_{A}(L)=L\left(s_{A}\right)-\sum_{r=0}^{\infty} L\left(a^{\prime r}\right)$ and 
$t_{r}=L\left(a^{\prime r}\right)$. Conversely any functional of this form is B-linear on $(A)$,

(iii) if $L(x)$ is a B-linear functional on $(A)$ with $t_{A}(L) \neq 0$ there is a normal matrix $D$ with $(D)=(A), D(x)=L(x)$ for $x \in(A)$.

These results are given in Mazur [2], Theorem 1, p. 44, Theorem 2, p. 45 , respectively. The condition $t \neq 0$ in (iii) will be shown necessary as a corollary to Theorem 2.1.1. Using the method of Banach $[1, \mathrm{p} .66]$ it follows that

$$
\|L\|=|t|+\sum_{r=0}^{\infty}\left|t_{r}\right|
$$

where, as usual, $\|L\|$ is the upper bound of $|L(x)|$ on the unit sphere in $(A)$.

2. Co-regular and co-null matrices.

Definition. Let $A$ be an arbitrary conservative matrix, $L(x)$ a B-linear functional on $(A)$. We define $\rho(L)=L(\dot{\mathrm{i}})-\sum_{r=0}^{\infty} L\left(\delta^{r}\right)$.

The series converges absolutely for, by Lemma 1.1.1, $L(x)$ is continuous on $(I)$ using the metric of $(I)$ and we may apply Mazur's theorem (ii) since $I$ is normal.

Since in particular $A(x)$ is continuous on $(A)$ by the very definition of the metric, we have defined $\rho(A)$.We shall call a conservative matrix $A$ conservative $\rho$, where $\rho=\rho(A)$. Clearly a multiplicative $\rho$ matrix is conservative $\rho$.

Definition. $A$ conservative matrix $A$, whether normal or not, will be called co-regular if $\rho(A) \neq 0$, co-null if $\rho(A)=0$.

A regular matrix is co-regular. A multiplicative zero matrix is co-null. Notice that $\rho(A)=\lim _{n \rightarrow \infty} \sum_{k=0}^{\infty} a_{n k}-\sum_{k=0}^{\infty} \lim _{n \rightarrow \infty} a_{n k}$.

In all extensions to conservative matrices which are carried out here, the following identity is fundamental. The number $t_{A}(L)$ has been defined in the statement of Mazur's theorem (ii).

TheOREM 2.0.1. If $A$ is a normal, conservative matrix and $L(x)$ is a B-linear functional on $(A)$, then $\rho(A) \cdot t_{A}(L)=\rho(L)$.

We have, by Mazur's theorem (ii) with $t=t_{A}(L), L(x)=t A(x)$ $+\sum_{r=0}^{\infty} t_{r} A_{r}(x)$. From this follows

$$
\begin{aligned}
L(\mathrm{i})-t A(\mathrm{i}) & =\sum_{r=0}^{\infty} t_{r} \sum_{k=0}^{r} a_{r k} \\
& =\sum_{k=0}^{\infty} \sum_{r=k}^{\infty} t_{r} A_{r}\left(\delta^{k}\right) \\
& =\sum_{k=0}^{\infty}\left[L\left(\delta^{k}\right)-t A\left(\delta^{k}\right)\right]
\end{aligned}
$$

which immediately gives the required result. The inversion in the second 
step is justified since

$$
\sum_{r}\left|t_{r}\right| \sum_{k}\left|a_{r k}\right| \leqq\|A\| \sum\left|t_{r}\right|<\infty .
$$

ThEOREM 2.0.2. If $A, B$ are conservative, $A$ is normal, and $B$ is row-finite then $\rho(B A)=\rho(B) \rho(A)$. In particular if both are normal $\rho(A B)=\rho(B A)$.

(We say that $B$ is row-finite if $b_{n k}=0$ for sufficiently large $k$, depending on $n$.) Let $C=B A$. The hypotheses imply associativity of matrix multiplication in the sense that $C x=A(B x)$ if $x$ is a column vector. Since $C A^{-1}=B$ is conservative we have $(C) \supseteq(A)$. By Mazur's theorem (i) $C(x)$ is a $B$ linear functional on $(A)$ and we have $t_{A}(C)=C\left(s_{A}\right)-\sum_{r=0}^{\infty} C\left(a^{\prime r}\right)=B(\mathrm{i})$ $-\sum_{r=0}^{\infty} B\left(\delta^{r}\right)=\rho(B)$ and the result follows from Theorem 2.0.1.

We now have a result showing that co-null matrices sum "more" sequences than co-regular matrices. We shall say that $A$ is not weaker than $B$ if $(A) \supseteq(B)$, weaker than $B$ if $(A) \subset(B)$.

TheOREM 2.0.3. If $A, B$ are conservative matrices with $A$ normal, $(A) \subseteq(B)$, then if $A$ is co-null so is $B$. ( $A$ co-regular matrix cannot be not weaker than a normal co-null matrix.)

Setting, in Theorem 2.0.1, $L(x)=B(x)$ we have $\rho(A) t_{A}(B)=\rho(B)$. The result follows from this.

Since, as in Theorem 2.0.2, $t_{A}(B)=\rho\left(B A^{-1}\right)$, given a normal matrix $A$ we may choose any normal co-null matrix $C$, set $B=C A$, and we shall have $(B) \supset(A), B$ co-null. Thus any normal co-regular matrix is weaker than some co-null matrix. That $(B) \neq(A)$ here follows from the following corollary.

CoROllary 1. A normal co-null matrix is stronger than convergence, that is, it must sum divergent sequences.

For multiplicative matrices this result is due to Agnew [3]. It follows from Theorem 2.0.3 since $\rho(I)=1$ and so a normal co-null matrix cannot have $(I)$ as its field. (Agnew obtained the result constructively.)

CoRollary 2 (Invariance). If two normal, conservative matrices $A, B$ have $(A)=(B)$, then they are co-regular or co-null together.

Thus it appears that the adjectives co-regular and co-null can be applied directly to summability fields.

THEOREM 2.0.4. A co-regular matrix cannot sum every bounded sequence.

Steinhaus [1] has proved this for regular matrices. A proof is given by Agnew $[2$, p. 596]. Let $A$ be the matrix. Let $B$ be the matrix defined by $b_{n k}=a_{n k}-a_{k}$ where $a_{k}=\lim _{n \rightarrow \infty} a_{n k}$. Applying Mazur's theorems (i) and (ii) on $(I)$ we have $A(x)=\rho(A) I(x)+\sum_{r=0}^{\infty} a_{r} x_{r}$ for $x \in(I)$ and so $B(x)=\rho(A) I(x)$ so that $B$ is multiplicative $\rho(A)$, and, using (1.1.1), sums exactly the sam $\epsilon$ 
bounded sequences that $A$ does. Thus, if $\rho \neq 0, \rho^{-1} B$ is regular and our result follows from that of Steinhaus. This simple type of reasoning can be applied to give the generalization of several results, for instance that of Agnew [3, p. 99] that the bounded field of a multiplicative matrix is non-separable (using a certain metric) or else contains only convergent sequences.

2.1. Invariance of $t$. We have defined $t_{A}(L)=L(s)-\sum L\left(a^{\prime r}\right)$ for Mazur's theorems. Its importance is illustrated there in parts (ii) and (iii).

THEOREM 2.1.1 (Invariance of $t$ ). If $A, B$ are normal, conservative matrices with $(A)=(B)$ and if $L(x)$ is a B-linear functional on either field (thus on both), then $t_{A}(L)$ and $t_{B}(L)$ are both zero or both not zero.

Lemma 1.1.1 justifies the parenthesized phrase. Remembering that $B(x)$ is a $B$-linear functional on $(A)$ and vice versa we have, using the results of Mazur [(2) pp. 42, 43], and setting $C=B A^{-1}$ (the identities may be checked merely by evaluation of both sides), $s_{A}=B\left(s_{A}\right) s_{B}+\sum_{r=0}^{\infty}\left[B_{r}\left(s_{A}\right)-B\left(s_{A}\right)\right] b^{\prime r}$ $=C(\mathrm{i}) s_{B}+\sum_{r=0}^{\infty}\left[C_{r}(\mathrm{i})-C(\mathrm{i})\right] b^{\prime r}$, and $a^{\prime p}=c_{p} s_{B}+\sum_{r=0}^{\infty}\left(c_{r p}-c_{p}\right) b^{\prime r}$ where $c_{p}=C\left(\delta^{p}\right)$. Thus $t_{A}(L)=L\left(s_{B}\right)\left[C(\mathrm{i})-\sum_{p=0}^{\infty} c_{p}\right]+\sum_{r=0}^{\infty} L\left(b^{\prime r}\right)\left\{\left[C_{r}(\mathrm{i})-C(\mathrm{i})\right]\right.$ $\left.-\sum_{p=0}^{\infty}\left(c_{r p}-c_{p}\right)\right\}=L\left(s_{B}\right) \rho(C)+\sum_{r=0}^{\infty} L\left(b^{\prime r}\right)\left[-C(\mathrm{i})+\sum_{p=0}^{\infty} c_{p}\right]=\rho(C) t_{B}(L)$ or

$$
t_{A}(L)=\rho(C) t_{B}(L)
$$

This result is immediate without computation in case $\rho(A) \neq 0$, from Theorem 2.0.2. Thus if $t_{B}(L)$ is 0 the same is true for $t_{A}(L)$.

Corollary. The condition $t \neq 0$ is necessary in Mazur's theorem (ii).

This follows by setting $L(x)=B(x)$ in Theorem 2.1.1, noting that $t_{B}(B)=1$. The same result follows from the fact that $t_{A}(B)=\rho\left(B A^{-1}\right)$ and so, using Theorem 2.0.2,

$$
t_{A}(B) t_{B}(A)=1
$$

3. Invariance of type $M$. In the course of this section all the results of Banach [1, pp. 91-95] are shown to apply to conservative matrices. Mazur, Hill, and others have used the concept type $M$, defined as follows. $A$ sequence $\left\{\alpha_{r}\right\}$ such that

$$
\begin{aligned}
& \sum_{r=0}^{\infty}\left|\alpha_{r}\right|<\infty, \\
& \sum_{r=0}^{\infty} \alpha_{r} a_{r k}=0
\end{aligned}
$$

for each $k$

is said to be orthogonal to the matrix $A$. Moreover, if the only sequence orthogonal to $A$ is identically zero $A$ is said to be of type $M$.

3.1. The orthogonal functionals. Invariance. Let $\left\{\alpha_{r}\right\}$ be orthogonal to a 
normal, conservative matrix $A$ and set $\alpha(x)=\sum_{r=0}^{\infty} \alpha_{r} A_{r}(x)$. We call $\alpha(x)$ an orthogonal functional. By Mazur's theorem (ii), $\alpha(x)$ is a $B$-linear functional on $(A)$ with $t(\alpha)=0$.

THEOREM 3.1.1 (Invariance). Let $A, B$ be normal, conservative matrices with $(A)=(B)$. If $L(x)$ is an orthogonal functional on $(A)$ then it is an orthogonal functional on $(B)$.

We have $L(x)=\sum \alpha_{r} A_{r}(x)$ with $\left\{\alpha_{r}\right\}$ orthogonal to $A$. As mentioned above $t_{A}(L)=0$ and so by Theorem 2.1.1 $t_{B}(L)=0$. This means, by Mazur's theorem (ii), taking account of Lemma 1.1.1. that $L(x)=\sum \beta_{r} B_{r}(x)$ with $\sum\left|\beta_{r}\right|<\infty$. Now, for each $p, 0=\sum \alpha_{r} a_{r p}=L\left(\delta^{p}\right)=\sum \beta_{r} b_{r p}$. The result follows.

Incidentally, setting $x=a^{\prime p}$ in the two expressions for $L(x)$ we have

$$
\alpha_{p}=\sum_{r=p}^{\infty} \beta_{r} c_{r p}, \quad p=0,1,2, \cdots,
$$

where $C=B A^{-1}$.

CoRollary (Invariance of type $M$ ). If $A, B$ are normal, conservative matrices with $(A)=(B)$, then both matrices are of type $M$ or neither is.

Suppose that $A$ is of type $M$. Then there is no orthogonal functional on $(A)$ except the identically zero functional. The same is then true for $B$ by Theorem 3.1.1.

Recalling the definition of $F$ in 1.1 we have

LEMMA 3.1.2. For a normal co-regular matrix $A$, every $B$-linear functional $L$ on $(A)$ which vanishes on $F$ is an orthogonal functional.

Immediately, $\rho(L)=0$ and so by Theorem 2.0.1 $t(L)=0$ and $L(x)$ $=\sum \alpha_{r} A_{r}(x)$. The result follows since $L\left(\delta^{p}\right)=0$ as in Theorem 3.1.1.

3.2. We now state some generalizations of results previously known for regular or multiplicative matrices (Banach $[1, \mathrm{pp} .91-95]$ ) with an indication of proof where the proofs differ from those of Banach.

Theorem 3.2.1. If $A$ is a normal, conservative matrix then

(a) every orthogonal functional on $(A)$ vanishes on $(A)_{0}$, the set of bounded sequences in $(A)$,

(b) if ${\overline{(A)_{0}}}_{0}(A)$ then $A$ is of type $M$. If in addition $A$ is co-regular, then

(c) $\overline{(I)}=\overline{(A)}_{0}$,

(d) $A$ is of type $M$ if and only if $\overline{(I)}=(A)$,

(e) if $x \in(A)$, every orthogonal functional vanishes at $x$ if and only if $x \in \overline{(I)}$,

(f) if $(A)$ contains a bounded divergent sequence $(I)$ is not closed in $(A)$.

The closures indicated are taken with respect to the metric of $(A)$. Part (f) follows immediately from (c). 
Proof of (d). If $\overline{(I)} \neq(A)$ there exists a $B$-linear functional which vanishes on $(I)$ but is not identically zero. By Lemma 3.1.2 it is an orthogonal functional and so $A$ is not of type $M$. If on the other hand $(\bar{I})=(A)$ the result follows from (c) and (b). The result is false for co-null matrices as may be seen by consideration of a multiplicative zero matrix $A$, for $A(x)=0$ on $(I)$ while $A\left(s_{A}\right)=1$, and yet such matrices may be of type $M$.

COROLlary. If $A$ is normal, conservative, then $\overline{(I)}$ includes the field of every co-regular matrix weaker than $A$ which is of type $M$.

This is an application of Lemma 1.1.1 to (d) above.

3.3. Consistency. One of the important applications of the idea of type $M$ is to consistency. Mazur [2, pp. 48-49 and Theorem 7], proves that a normal, regular matrix $A$ is consistent with every matrix $B$ such that (i) $(B) \supseteq(A)$, (ii) $B$ is regular, if and only if $A$ is of type $M$. We now provide the best possible generalization of this result to normal, conservative matrices. The set $F$ was defined in 1.1 .

THEOREM 3.3.1. A normal co-regular matrix $A$ is consistent with every matrix $B$ such that (i) $(B) \supseteq(A)$, (ii) $B$ agrees with $A$ on $F$, if and only if $A$ is of type $M$.

REMARKS. It will be clear that in the statement "every matrix $B$ " may be replaced by "every normal matrix $B$." For a multiplicative zero matrix $A$, $A(x)=2 A(x)=0$ on $F$ but $A$ and $2 A$ disagree at $s_{A}$ so that the theorem fails for co-null matrices.

Proof. If $A$ is of type $M,(B) \supseteq(A), B$ agreeing with $A$ on $F$, consider $B(x)-A(x)$ on $(A)$. By Lemma 3.1.2 it is an orthogonal functional and so is identically zero. If on the other hand $A$ is not of type $M$, there exists, by Theorem 3.2.1 (d), a $B$-linear functional $G(x)$ which is zero on $(I)$ but not identically zero, that is, an orthogonal functional by Lemma 3.1.2. Setting $L(x)=A(x)+G(x)$ we have $\rho(L)=\rho(A) \neq 0$ and, from Theorem 2.0.1, $t_{A}(L)$ $\neq 0$. This allows us to apply Mazur's theorem (iii) to conclude the existence of a normal matrix $B$ with $(B)=(A), B(x)=L(x)$. Evidently $B$ agrees with $A$ on $F$ but not everywhere.

The following generalization in another direction has interesting special cases. Let $F_{D}$ denote the set $\left\{s_{D}, d^{\prime k}\right\}$ where $D$ is a normal matrix.

CoRollary. If $D$ is a normal matrix and $A$ is a normal co-regular matrix with $(A) \supseteq(D)$, then $A$ is consistent with every matrix $B$ such that $(B) \supseteq(A)$ and $B$ agrees with $A$ on $F_{D}$ if and only if $A D^{-1}$ is of type $M$.

The special cases $D=A,(D)=(I)$ are interesting as well as others about to be considered. Setting $D=I$ we have Theorem 3.3.1.

3.31. Subregions of $(I)$. Consider again Theorem 3.3.1. The set $F$ is, so to speak, representative of $(I)$ and could be replaced by $(I)$ in the statement. 
Now for $n=0,1,2, \cdots$ consider the matrix $A^{-n}$ where $A$ is normal, conservative. Since $A^{-n} \cdot A^{n+1}=A$ is conservative, $\left\{\left(A^{-n}\right)\right\}$ is a monotonely decreasing sequence of subregions of $(I)$. That $\prod\left(A^{-n}\right)$ need not be a single point (sequence) is shown by taking $A$ to be the first Cesàro mean, in which case the product set contains $F$. For positive integral $m, A^{m}$ is of type $M$ if and only if $A$ is, see for instance Hill [1, Theorems 5 and 6, p. 704]. Thus the corollary to Theorem 3.3.1 gives the following result which is stronger than Theorem 3.3.1.

THEOREM 3.31.1. A normal co-regular matrix $A$ is consistent with every $B$ such that $(B) \supseteq(A)$ and $B$ agrees with $A$ on any one of $\left(A^{-n}\right), n=0,1,2, \cdots$, if and only if $A$ is of type $M$.

We remark also that by Theorem 3.2.1 (d) and the above, the density of any one of $\left(A^{-n}\right)$ in $(A)$ is equivalent to $A$ 's being of type $M$, where $A$ is normal, co-regular.

These subregions of $(I)$ play a role also in the determination of $\rho(L)$. The behaviour of $L(x)$ on $(I)$ determines $\rho(L)$ but we can, at least for coregular matrices, determine $\rho(L)$ from the behaviour of $L(x)$ on any one of the subregions $\left(A^{-n}\right)$ of $(I)$.

Lemma 3.31.2. If $A$ is a normal, conservative matrix, $L(x)$ is a B-linear functional on $(I), m$ is a non-negative integer, and $E=A^{-m}$, then $t_{E}(L)=\rho(L)$ $[\rho(A)]^{m}$.

By Lemma 1.1.1 the condition on $L$ is satisfied if, for instance, $L$ is $B$-linear on $(A)$. To obtain the formula we define the matrix $C$ by $c_{n k}=L\left(\delta^{k}\right)$ for $k \leqq n-1, c_{n n}=\rho(L), c_{n k}=0$ for $k>n$. Then $C$ is a triangular conservative matrix. Set $D=C A^{m}=C E^{-1}$. Then $\rho(D)=\rho(C)[\rho(A)]^{m}$ by Theorem 2.0.2. However $\rho(D)=D(\mathrm{i})-\sum D\left(\delta^{r}\right)=C\left(s_{E}\right)-\sum C\left(e^{\prime r}\right)=t_{E}(C)$. Combining these identities with $C(x)=\lim \sum_{k=0}^{n} \quad c_{n k} x_{k}=\lim \left\{\rho(L) x_{n}\right.$ $\left.+\sum_{k=0}^{n-1} L\left(\delta^{k}\right) x_{k}\right\}=\rho(L) I(x)+\sum_{k=0}^{\infty} L\left(\delta^{k}\right) x_{k}=L(x)$ for $x \in(I)$ by Mazur's theorem (ii), we have the result noting that $s_{E}$ and every $e^{\prime r}$ belong to $(I)$ since $A^{m}$ is conservative.

3.32. Characterization of type $M$ by co-ordinate systems. The above ideas are useful in proving the results of this section although they are not essential and the theorem can be proved without resort to Mazur's theorem (iii).

We shall say that a set $H$ of sequences forms a base for a set $K$ if to every member $x$ of $K$ and every positive $\epsilon$ there corresponds a finite linear combination $y$ of members of $H$ such that $\left|x_{n}-y_{n}\right|<\epsilon$ for all $n$, where $x=\left\{x_{n}\right\}$, $y=\left\{y_{n}\right\}$. For our purposes we shall take the closed linear hull of $H$ in the sense of this metric, that is, the smallest set of sequences for which $H$ forms a base.

THEOREM 3.32.1. A normal co-regular matrix $A$ is of type $M$ if and only if 
the set of sequences formed by the columns of $A$ and the sequence $\left\{\sum_{k=0}^{n} a_{n k}\right\}$ forms a base for $(I)$.

We require a lemma.

LEMMA 3.32.2. Let a set $H$ of convergent sequences form a base for $(I)$ and let $C$ be a conservative matrix. The matrix $C$ is regular if and only if it sums each member of $H$ to its ordinary limit.

The condition is clearly necessary. Now suppose that $C$ sums each member of $H$ to its ordinary limit and let $y$ be any convergent sequence. By hypothesis $y$ can be written in the form $y_{n}=\lim _{p \rightarrow \infty} \sum_{r=0}^{m(p)} y_{r}^{p} z_{n}^{r, p}$ where $y_{r}^{p}$ is a number, $z^{r, p} \in H, z_{n}^{r, p}$ is its $n$th term, and $m(p)<\infty$. Moreover the limit is uniform in $n$.

Secondly $C(x)$ is a $B$-linear functional on $(I)$ by Mazur's theorem (i). Using these two facts we have $C(y)=\lim _{p} C\left(\sum_{r} y_{r}^{p} z^{r, p}\right)=\lim _{p} \sum_{r} y_{r}^{p} C\left(z^{r, p}\right)$ $=\lim _{p} \sum_{r} y_{r}^{p}\left(\lim _{n} z_{n}^{r, p}\right)=\lim _{n} \lim _{p} \sum_{r} y_{r}^{p} z_{n}^{r, p}=\lim _{n} y_{n}$ and so $C$ is regular.

Proof of Theorem 3.32.1. Calling the sequences mentioned in the statement $a^{k}$ (the $k$ th column of $A$ ) and $S$ respectively, suppose they form a base for $(I)$. If $B$ is a normal matrix with $(B) \supseteq(A)$ agreeing with $A$ on $F$, set $C=B A^{-1}$ so that $C$ is a normal, conservative matrix. We have, for each $k, C\left(a^{k}\right)=B\left(\delta^{k}\right)=A\left(\delta^{k}\right)=I\left(a^{k}\right)$ and $C(S)=B(\mathrm{i})=A(\mathrm{i})=I(S)$ since $B$ agrees with $A$ on $F$. By the lemma, $C$ is regular. Thus $B$ is consistent with $A$. By Theorem 3.3.1 and the remark on that theorem, $A$ is of type $M$.

Next suppose that the set mentioned does not form a base for $(I)$. Let $H$ be the closed linear hull of this set taken as mentioned above. By supposition $H$ is a proper subset of $(I)$. Let $y \in(I)-H$. Now, working in the space $(I)$ with its metric, since $H$ is closed there is a $B$-linear functional $G(x)$ defined on $(I)$ which is zero on $H$ and one at $y$. Now set $L(x)=G(x)+\lim x_{n}$ for $x \in(I)$. Since $G(x)$ vanishes on $H$ we have $G(S)-\sum G\left(a^{k}\right)=0$ and thus, by Lemma 3.31.2 with $m=1, \rho(G)=0$, and so $\rho(L)=1$. By Mazur's theorem (iii) there is a normal matrix $C$ with $(C)=(I), C(x)=L(x)$. Setting $B=C A$ we can easily verify that $(B)=(A)$ and $B$ agrees with $A$ on $F$. For instance $B(\mathrm{i})=C(S)=L(S)=\lim S_{n}=A(\mathrm{i})$. However if we set $z=A^{-1} y$ so that $z \in(A)$ we have $B(z)=C(y)=1+\lim y_{n}=1+A(z)$. By Theorem 3.3.1 $A$ is not of type $M$.

\section{Miscellaneous.}

THEOREM 4.0.1. If $A$ is a normal, conservative matrix which sums a divergent sequence then $(I)$ is of first category in $(A)$.

We have seen that $(I)$ may be dense in $(A)$ in Theorem 3.2.1 (d). We map (A) into itself by associating $A x \in(I)$ with $x \in(A)$. This mapping is continuous since if the sequence $\left\{x^{r}\right\}$ of elements of $(A)$ converges to $x$, this means by definition that $\left\{A x^{r}\right\}$ converges to the sequence $A x$ using the metric of $(I)$ and so, a fortiori (Lemma 1.1.1), using the metric of $A$. The mapping is 
also one-to-one since $A$ is normal. The result follows by Theorem 3, p. 38 in Banach [1].

4.1. A problem. We can state the following result for co-regular matrices. Suppose that $L(x)$ is a $B$-linear functional on $(A)$, where $A$ is normal, coregular. Then the necessary and sufficient condition that there be a matrix $B$ with $(B)=(A), B(x)=L(x)$ is $\rho(L) \neq 0$. This follows from Mazur's theorem (iii), the corollary to Theorem 2.1.1, and Theorem 2.0.1. The notable part of this result is that $\rho(L)$ is determined purely by the behaviour of $L$ on $(I)$. However this characterization fails for co-null matrices. Indeed $\rho=0$ for all $B$-linear functionals on $(A)$ if $A$ is normal, co-null. It is then interesting to ask whether such a characterization for a functional on a co-null field in terms only of its behaviour on $(I)$ can be found.

\section{BIBLIOGRAPHY}

R. P. AgNew

1. Ranges of inconsistency of regular transformations and allied topics, Ann. of Math. (2) vol. 32 (1931) pp. 715-722.

2. Summability of subsequences, Bull. Amer. Math. Soc. vol. 50 (1944) pp. 596-598.

3. Convergence fields of methods of summability, Ann. of Math. (2) vol. 46 (1945) pp. 93-101. S. BANACH

1. Théorie des opérations linéaires, Warsaw, 1932.

A. L. BRUDNo

1. Summation of bounded sequences by matrices (in Russian), Rec. Math. (Mat. Sbornik) N.S. vol. 16 (1945) pp. 191-247.

J. D. HiLI.

1. On perfect methods of summability, Duke Math. J. vol. 3 (1937) pp. 702-714.

S. MAZUR

1. Über lineare Limitierungsverfahren, Math. Zeit. vol. 28 (1928) pp. 599-611.

2. Eine Anwendung der Theorie der Operationen bei der Untersuchung der Toeplitzschen Limitierungsverfahren (Prize-winning paper), Studia Mathematica vol. 2 (1930) pp. 4050.

H. Steinhaus

1. Some remarks on the generalization of the notion of limit (in Polish), Prace Matematycznofizyczne vol. 22 (1911) pp. 121-134.

Brown UNIVERSITY,

Providence, R.I. 